\title{
The Mini Cog had high sensitivity and specificity for diagnosing dementia in community dwelling older adults
}

Scanlan J, Borson S. The mini-cog: receiver operating characteristics with expert and näive raters. Int J Geriatr Psychiatry $2001 \mathrm{Feb} ; 16: 216-22$.

QUESTION: How accurate is the Mini Cog as a diagnostic screening test for dementia in a community dwelling elderly population compared with classification with the Consortium to Establish a Registry for Alzheimer's Disease (CERAD), Diagnostic and Statistical Manual of Mental Disorders, 4th edition (DSM-IV), and the National Institute of Neurological and Communicative Disorders and Stroke and the Alzheimer's Disease and Related Disorders Associations (NINCDS-ADRDA) (the diagnostic standards) and with 2 other diagnostic screening tests, the Mini Mental State Examination (MMSE) and the Cognitive Abilities Screening Instrument (CASI)?

\section{Design}

\{Blinded comparison $\} *$ of the Mini Cog with the CERAD, DSM-IV, and NINCDS-ADRDA and the MMSE and CASI.

\section{Setting}

Community based study in Seattle, Washington, USA.

\section{Participants}

249 community dwelling older adults (69\% women) who reflected the 5 major ethnic groups in the US. Participants with uncertain or very mild cognitive impairment (Clinical Dementia Rating of 0.5) were excluded.

\section{Description of test and diagnostic standards}

The Mini Cog combines the 3 item recall and the Clock Drawing Test (CDT). Uncued recall was used in deriving a score for the 3 item recall. For the CDT, participants were instructed to draw a large circle, fill in the numbers on a clock face, and set the hands at 8:20, with no time limit to complete the task. The CDT was scored by 2 independent markers $(0=$ no errors, $1-3=$ increasingly abnormal CDT). A series of Mini Cog scoring algorithms were tested by using various combinations of the 3 item recall and the CDT to construct receiver operating characteristic curves. Participants were classified as probably demented or probably not demented based on informants' history of cognitive decline (CERAD expanded history) and current functioning. This classification was confirmed using the CERAD, DSM-IV, and NINCDS-ADRDA.

\section{Main outcome measures}

Sensitivity, specificity, and positive and negative likelihood ratios.

\section{Main results}

7 different Mini Cog scoring algorithms were tested. The best performing algorithm was when the participant was judged to be demented if either the 3 item recall score was 0 or the 3 item recall score was 1 to 2 and a CDT error score was rated between 1 (mild) and 3 (severe) (table). When this algorithm was used, the Mini Cog had the highest sensitivity (99\%) of the 3 instruments tested. The MMSE at conventional cut off points had a sensitivity of $91 \%$ and a specificity of $92 \%$. The CASI had comparable sensitivity but higher specificity than the MMSE.

\section{Conclusion}

A Mini Cog scoring algorithm combining scores from the 3 item recall and the Clock Drawing Test had high sensitivity and specificity for diagnosing dementia in community dwelling older adults.

*Information provided by author.

Operating characteristics of the Mini-Cog for detecting dementia in community dwelling older adults $\dagger$

\begin{tabular}{llll} 
Sensitivity & Specificity & +LR & -LR \\
\hline $99 \%$ & $93 \%$ & 14.1 & 0.01 \\
\hline
\end{tabular}

†Diagnostic terms defined in glossary; LRs calculated from data in article.
Sources of funding: National Institute on Ageing and Health Resources and Services Administration.

For correspondence: Dr I Scanlan, Box 356560, University of Washington School of Medicine, $1959 \mathrm{NE}$

Pacific Street, Seattle WA 98195-6560, USA. Fax +1206543 9520 .

\section{COMMENTARY}

The Mini Cog takes $<5$ minutes to complete, is unaffected by education or language, and can be scored by untrained markers with minimal loss of sensitivity and specificity. It incorporates the 2 strongest elements of the MMSE-testing of short-term memory and visuo spatial representation-while adding assessment of executive function. The high likelihood ratio seen with the best performing algorithm reflected a sample population of those with a diagnosis of dementia and will be lower in populations with a low prevalence of dementia.

The efficiency and practicality of the Mini Cog make it suitable for primary care clinicians. The traditional goal of dementia screening has been to identify patients and families in need of educational and psychosocial interventions. Increasingly, screening is aimed at early identification of Alzheimer's disease (AD) to implement treatments that slow cognitive decline and prolong functional independence. The prevalence of dementia increases exponentially from $<1 \%$ at 60 years of age to $>10 \%$ for people $75-79$ years of age, ${ }^{1}$ making 75 years of age appropriate to begin routine screening. On the basis of the rising annual incidence of dementia with older age groups, ${ }^{2}$ patients between 75 and 79 years of age without dementia should be rescreened within 5 years, and patients $\geqslant 80$ years of age should be rescreened every 2 years. The sensitivity of screening may be enhanced by giving informers a short questionnaire like the Informant Questionnaire on Cognitive Decline in the Elderly (IQCODE). ${ }^{3}$ A normal Mini Cog and IQCODE effectively rule out dementia but not the pre-AD state of mild cognitive impairment. ${ }^{2}$ In patients complaining of memory problems, a normal Mini Cog warrants additional assessment. A positive Mini Cog should be followed by a full MMSE. Calvin Hirsch, MD University of California at Davis Sacramento, California, USA

1 Kukull WA, Ganguli M. Epidemiology of dementia: concepts and overview. Neurol Clin 2000;18:923-50.

2 Petersen RC, Stevens JC, Ganguli M, et al. Practice parameter: early detection of dementia: mild cognitive impairment (an evidence-based review). Report of the quality standards subcommittee of the American Academy of Neurology. Neurology 2001;56:1133-42.

3 Jorm AF. A short form of the informant questionnaire on cognitive decline in the elderly (IQCODE): development and cross-validation. Psychol Med 1994;24:145-53. 